\title{
Poliposis adenomatosa familiar y prevención del cáncer colorrectal: reporte de un caso
}

\section{Familial adenomatous polyposis and colorectal cancer prevention: A case report}

Axel Duval C., ${ }^{*}{ }^{*}$ Jhosep Fernández B. ${ }^{2}$

1 Médico Cirujano. Interno de Investigación del Centro de Investigación Radiológica, Universidad de Columbia. Nueva York, Estados Unidos.

2 Médico Cirujano. Interno de Posgrado del Hospital Dr. Miguel Pérez Carreño, Instituto Venezolano de los Seguros Sociales (IVSS). Caracas, Venezuela.

*Correspondencia: ax.duval@gmail.com.

\section{INTRODUCCIÓN}

La poliposis adenomatosa familiar (PAF) es una enfermedad de transmisión hereditaria con un patrón autosómico dominante (1), caracterizada por un defecto del gen APC

\begin{abstract}
Resumen
La poliposis adenomatosa familiar (PAF) es una enfermedad hereditaria caracterizada por el crecimiento de múltiples adenomas epiteliales de distribución colorrectal, de patrón autosómico dominante causado por el defecto del gen APC. La degeneración de cáncer colorrectal en estos pacientes se considera inevitable en caso de no recibir el manejo terapéutico adecuado.

Se presenta el caso de una paciente femenina de 25 años, quien acudió a consulta luego de presentar una modificación del patrón evacuatorio y dolor abdominal, sin antecedentes familiares asociados, por lo que se correlacionó con paraclínicos y se diagnosticó PAF, con la posterior implementación del manejo terapéutico. Se decidió hacer una revisión bibliográfica y actualización del tema resaltando los aspectos clínicos de reconocimiento de la enfermedad, así como las conductas a tomar en consideración para la prevención del cáncer en pacientes con PAF.
\end{abstract}

\section{Palabras clave}

Poliposis adenomatosa familiar, cáncer colorrectal, prevención.

\section{Abstract}

Familial adenomatous polyposis (FAP) is a hereditary disease characterized by the growth of multiple colorectal epithelial adenomas. It is an autosomal dominant disorder caused by an APC gene defect. Degeneration to colorectal cancer is considered unavoidable in these patients if they do not receive adequate therapeutic management.

We present the case of a 25 -year-old female patient consulted after a change in her evacuation pattern and abdominal pain. She had no relevant family history associated but based on results of paraclinical tests diagnosis of FAP was made for which therapeutic management was implemented. This is a case report with a literature review and update of the topic highlighting clinical issues related to recognition of the disease and issues that should be taken into consideration for the prevention of cancer in patients with FAP.

\section{Keywords}

Familial adenomatous polyposis, cannula. 
aproximadamente el 0,5\% de todos los cánceres colorrectales (4). La edad promedio de inicio es de 16 años (5) y la progresión al cáncer colorrectal (CCR) ocurre a la edad de 40-50 años con penetrancia casi completa (7).

La manifestación primaria de la enfermedad es la aparición de múltiples pólipos (cientos o miles) sobre la mucosa rectocolónica, duodenal y gástrica $(8,9)$; de estructura histológica glandular tubular, vellosa o mixta; esta no determina un factor predictor significativo para la aparición de cáncer, como sí resulta el tamaño de los pólipos (10). Suele presentar manifestaciones extracolónicas, como pólipos gástricos y del intestino delgado (1), y extraintestinales que incluyen hipertrofia congénita del epitelio pigmentario de la retina, fibrosis mesentérica difusa (tumores desmoides), osteomas del maxilar inferior (en el $90 \%$ de los casos), del cráneo y huesos largos (variante fenotípica descrita como síndrome de Gardner), y diversas anormalidades dentales (11). También se producen neoplasias del sistema nervioso central como meduloblastoma (síndrome de Turcot), de la glándula tiroides, del sistema hepatobiliar y de las glándulas suprarrenales $(7,8,12)$.

El abordaje diagnóstico más importante de PAF es mediante el tamizaje en pacientes con antecedentes familiares (13). Sin embargo, se cree que un tercio de todos los casos es debido a mutaciones de novo (4) y sin presentar antecedente familiar alguno, lo que determina un mayor reto diagnóstico con implicaciones negativas en el pronóstico. El tratamiento quirúrgico es el indicado (1) y está dirigido a evitar el desarrollo de CCR, cuyo riesgo de aparición es muy elevado (14), y es inevitable cuando la enfermedad sigue su curso natural en pacientes que no se someten a un manejo quirúrgico (15).

\section{PRESENTACIÓN DEL CASO}

Se presenta el caso de una paciente femenina de 25 años, quien manifestó cuadro clínico de 1 mes de evolución, caracterizado por evacuaciones con sangre, no diarreicas y sin moco, en número de una (1) por día, de predominio matutino. Concomitantemente, presentaba dolor abdominal generalizado postevacuatorio, tipo cólico, de fuerte intensidad y que cedía luego de aproximadamente 1 hora. Además, se le asociaron cambios en el patrón evacuatorio en cuanto a la frecuencia ( 3 evacuaciones/día que se modificaron a $1 /$ día).

\section{Antecedentes personales y familiares}

Diagnóstico endoscópico de gastritis crónica desde los 13 años, con hallazgo de pólipo sésil único. Una abuela falleció a los 69 años por cáncer de mama, una abuela estaba viva con diagnóstico de artritis reumatoide y la madre estaba viva con diagnóstico de fibroma uterino. La paciente negó todo tipo de antecedente familiar de CCR, pólipos o PAF.

\section{Examen físico}

Peso: $45 \mathrm{~kg}$, talla: $168 \mathrm{~cm}$, índice de masa corporal (IMC): 15,9. Abdomen plano, con ruidos hidroaéreos presentes, blando, depresible, doloroso a la palpación profunda en el hemiabdomen inferior, sin signos de irritación peritoneal ni visceromegalias. Tacto rectal: esfínter anal normotónico y normotérmico; paredes rectales con múltiples masas palpables, de diverso diámetro, no dolorosas; ampolla rectal con heces pastosas, punta de guante con escasa cantidad de sangre. El resto de evaluación resultó dentro de los límites normales.

\section{Paraclínicos realizados}

Se realizaron exámenes de laboratorio que abarcaban hemograma y química sanguínea, y se encontraron entre los parámetros normales. Además, se indicaron estudios de imagen como radiografía abdominal con uso de enema baritado y se evidenciaron alteraciones del marco colónico con acumulación de secreción (Figura 1). En la rectosigmoidoscopia se apreciaron múltiples pólipos $(>100)$ de diversos tamaños que oscilan entre $5 \mathrm{~mm}$ y $3 \mathrm{~cm}$, algunos pediculados y otros sésiles, de aspecto tubulovelloso, desde el colon ascendente distal hasta el recto (Figuras 2 y 3 ).

Se realizó una gastroduodenoscopia que mostró la presencia de punteado blanco algodonoso en la segunda porción del duodeno con múltiples lesiones polipoideas sésiles en el cuerpo y el fondo gástrico (Figura 4).

El estudio histopatológico de los hallazgos reportó:

- Mucosa del colon sigmoides: adenoma tubular con displasia de alto grado focal (10\%).

- Pólipos del colon transverso: adenoma tubulovelloso con predominio del componente adenomatoso, en el $80 \%$ del material examinado, con áreas focales de displasia de alto grado.

- Pólipos en el cuerpo gástrico: pólipos hiperplásicos de tipo oxíntico con dilatación de criptas glandulares e inflamación crónica de la mucosa.

Según la evidencia obtenida por la clínica y los estudios complementarios del paciente, se estableció el diagnóstico de PAF.

\section{Tratamiento y evolución}

Se indicó una colectomía total más resección de los dos tercios superiores del recto con anastomosis ileorrectal y en una primera intervención se dejó ileostomía de protección. Se obtuvo tejido ileal, colónico y rectal, cuyo análisis anato- 

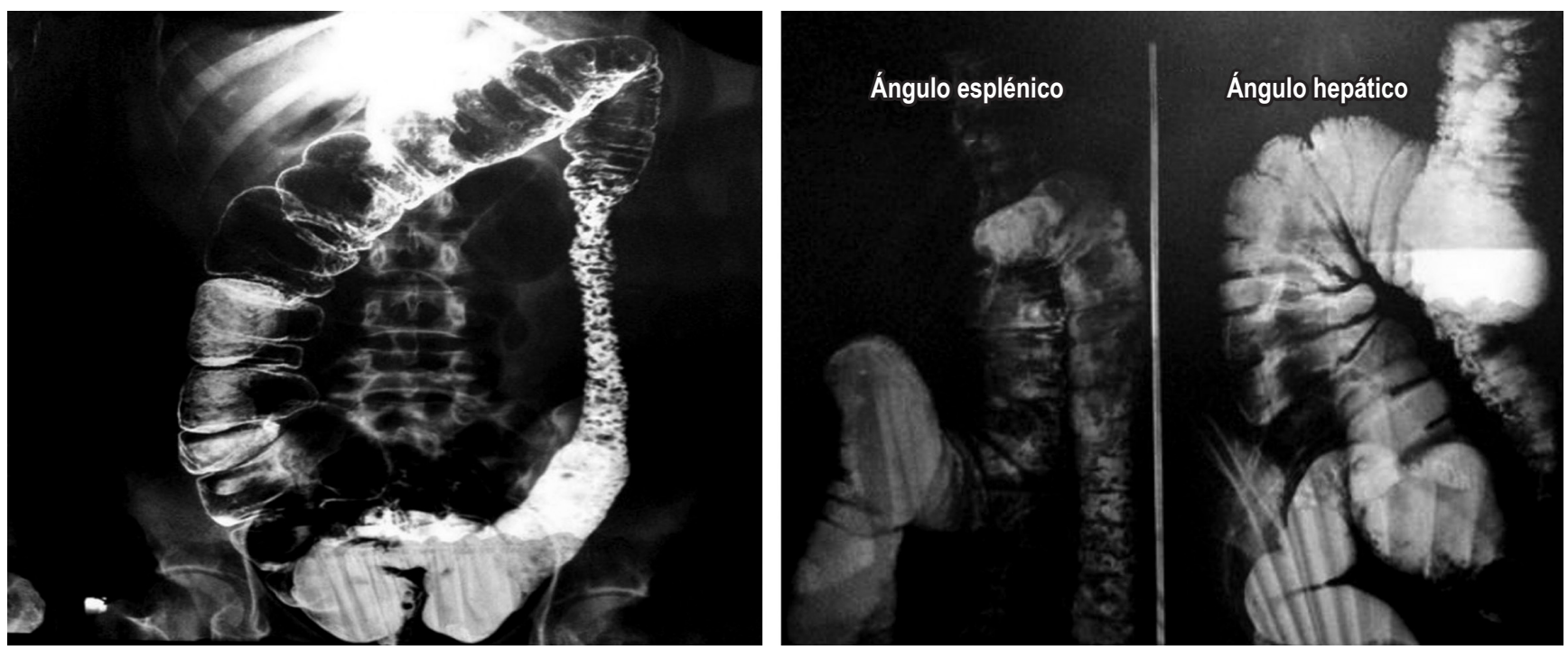

Figura 1. Radiografía abdominal en proyección anteroposterior, de pie, con uso de enema baritado. Se evidencia engrosamiento de las paredes colónicas, dilataciones irregulares del marco colónico, aumento y heterogeneidad de secreción en el colon ascendente y en el ángulo hepático, así como en el colon descendente y sigmoides con imágenes polipoideas múltiples y signos correlativos con úlceras en collar.

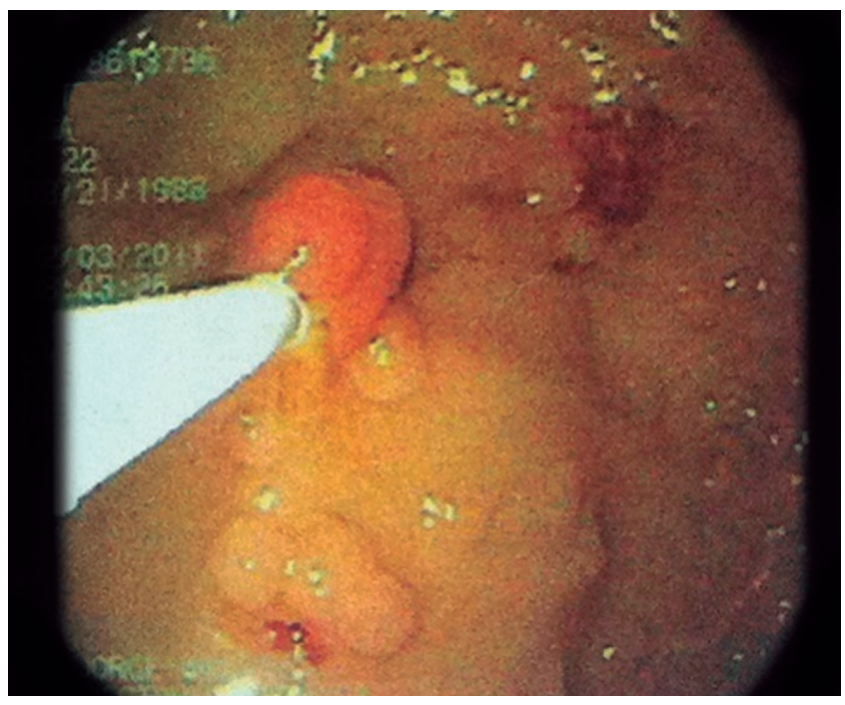

Figura 2. Rectosigmoidoscopia. Se evidencia un pólipo pediculado de aspecto tubular.

mopatológico reportó incontables estructuras polipoides en su mayoría constituidas por adenomas tubulovellosos, con evidencia de displasia de alto grado, pediculados y sésiles, asentados sobre la mucosa con cambios hiperplásicos multifocales. Dicha intervención evoluciona favorablemente.

Se reingresó a los 6 meses para cirugía programada de restitución terminoterminal, con complicación en el

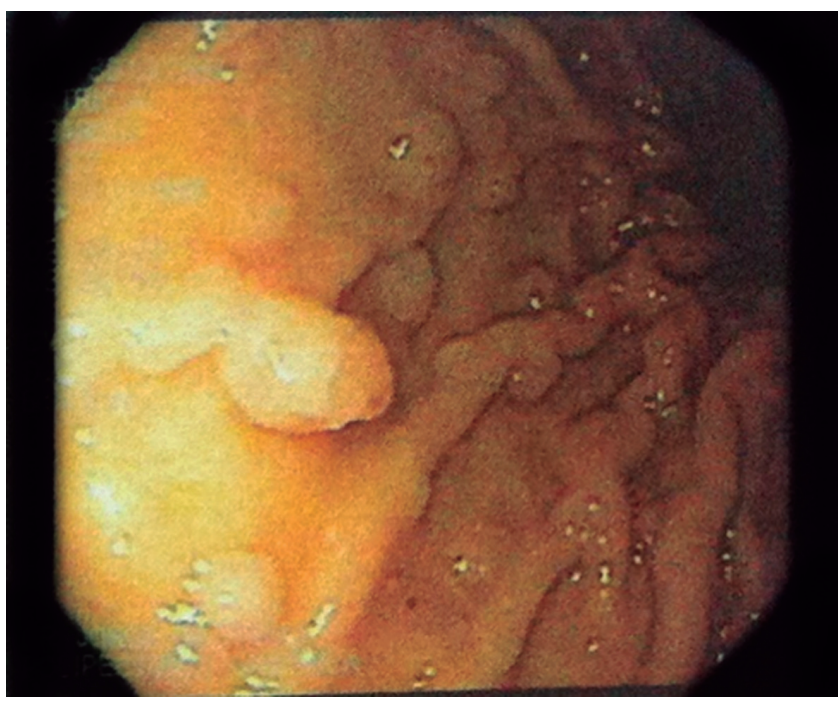

Figura 3. Rectosigmoidoscopia. Se evidencian múltiples pólipos sésiles, de aspecto velloso, de diámetros diversos.

postoperatorio inmediato de íleo paralítico, superado bajo tratamiento médico. En el año posterior a la intervención, presentó 3 episodios de obstrucción intestinal que ameritaron resolución quirúrgica. En el último episodio se evidenció un hallazgo de absceso intraabdominal en la fosa ilíaca y el flanco derecho que ameritó un manejo con bolsa de Bogotá, que requirió la hospitalización durante 8 

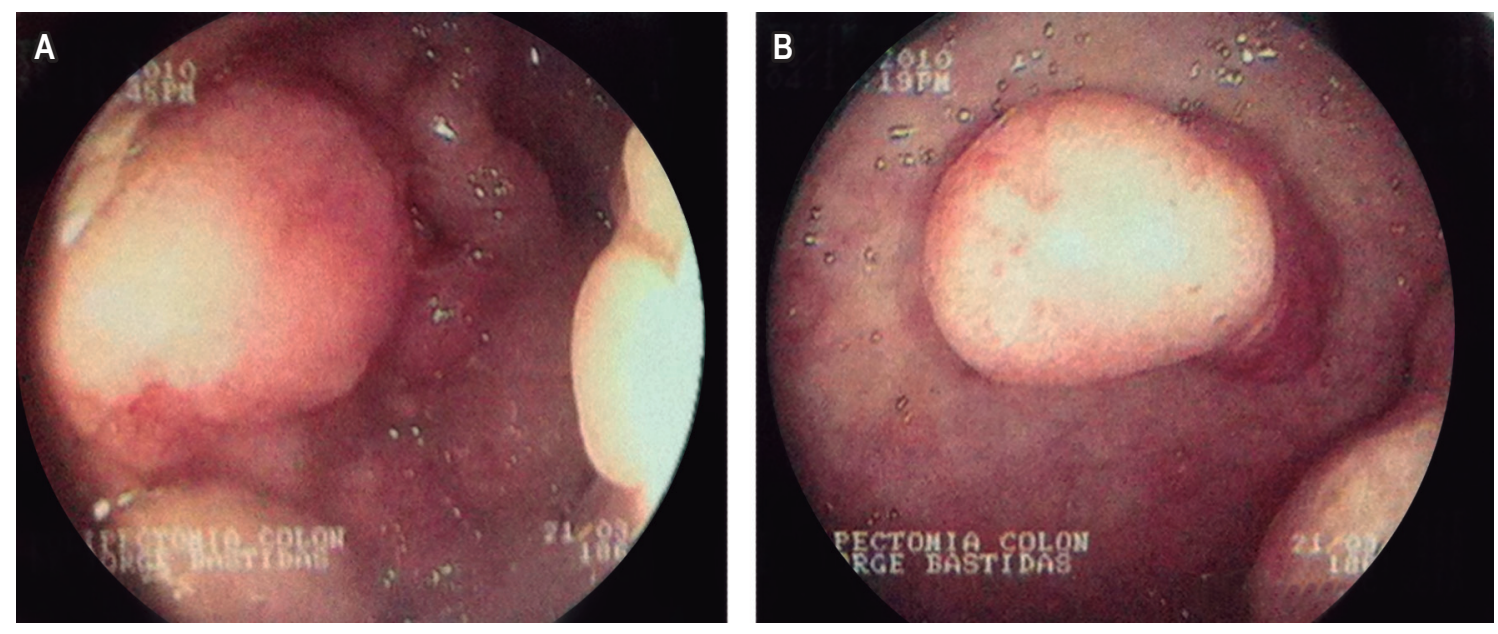

Figura 4. Gastroduodenoscopia. Fondo (A) y cuerpo (B) gástrico con múltiples pólipos sésiles de diámetros diversos.

meses con evolución favorable. Actualmente, la paciente se encuentra en buenas condiciones generales, con tránsito intestinal regular y sin ningún otro concomitante.

\section{DISCUSIÓN}

A pesar de que la PAF es una enfermedad estudiada y descrita, su prevención y diagnóstico precoz son los inconvenientes más comunes y decisivos para el pronóstico. En este punto radica la relevancia de esta revisión, considerando que la totalidad de los casos evolucionan en malignidad de no ser tratados (13). El diagnóstico se basa en aspectos clínicos y endoscópicos (12). La aparición progresiva de los pólipos deriva en la presencia de sangre en la materia fecal (visible o no) gracias principalmente a que los pólipos sangran hacia su estroma (8), que es el síntoma más común (1). Otros síntomas pueden ser flatulencia, cambios en el patrón evacuatorio o dolor postevacuatorio en el hemiabdomen inferior tipo cólico $(7,8)$. La exploración anorrectal puede evidenciar la presencia de pólipos si estos prolapsan a través del recto (1). Es también de gran importancia el interrogatorio que incluya los antecedentes familiares de pólipos colónicos y de cáncer de colon (15), tomando en cuenta que alrededor de un tercio de los pacientes no posee antecedente familiar relacionado alguno, como lo fue la paciente estudiada.

El diagnóstico se establece mediante criterios clínicos y se excluye por medio de un colon por enema con bario y aire, con la presencia de pólipos de naturaleza adenomatosa, o una colonoscopia total, la cual permite evidenciar lesiones de mayor tamaño y la toma de biopsia para descartar malignidad (16). Esto diferencia la enfermedad en su variación clásica ( $>100$ pólipos) o atenuada ( $<100$ pólipos) (7). Es necesaria la búsqueda de hallazgos extracolónicos, principalmente la presencia de pólipos duodenales (17) e hiperpigmentación epitelial de la retina, ya que esta última se observa en el $83 \%$ de las familias con PAF (7), así como la tamización de familiares y la determinación del gen APC $(2,14)$, a pesar de no haber sido posible el estudio genético en esta paciente por motivos socioeconómicos.

La cirugía es el pilar fundamental para el manejo de la PAF, en particular cuando existe una mutación entre los codones 1251 y 1309, los cuales expresan un fenotipo más grave (13). Con la individualización del estado de cada paciente y según su correlación genotipo-fenotipo existen opciones quirúrgicas que incluyen: proctocolectomía total con ileostomía permanente, colectomía con anastomosis ileorrectal (AIR) o proctocolectomía con anastomosis con bolsa ileoanal (IPAA). El primer abordaje no se realiza de manera profiláctica y es indicado en pacientes con poliposis rectal extensa, cáncer de la porción distal del recto o en los cuales la vigilancia pertinente se hace imposible. Por su parte, la colectomía AIR y la proctocolectomía IPAA son técnicas profilácticas llevadas a cabo en pacientes asintomáticos en riesgo, determinado por pruebas genéticas o colonoscopias predictivas. Sin embargo, el riesgo de desarrollar cáncer en la porción rectal conservada luego de 20 años es del $25 \%$, por lo que se requiere vigilancia periódica particularmente estricta $(13,18)$.

En pacientes con variante atenuada, de fácil seguimiento y con menos de 20 pólipos rectales cuyo tamaño sea $<5$ $\mathrm{mm}$, se considera el tratamiento médico $(19,20)$. El uso de antiinflamatorios no esteroideos (AINE) como sulindac $y$ celecoxib ha demostrado tener la mayor eficiencia para 
reducir la cantidad y el tamaño de los adenomas colorrectales caracterizados por los altos niveles de expresión de ciclooxigenasa 2 (COX-2) $(20,21)$. Su uso es aceptado como terapia adyuvante al tratamiento quirúrgico mas no como alternativa a este (22). La resolución quirúrgica es controvertida en pacientes portadores asintomáticos para los cuales, sin embargo, se recomienda la intervención al final de la adolescencia (14).

En individuos en riesgo, con evidencia de poliposis sin PAF o variante atenuada se sugiere la colonoscopia anual o una sigmoidoscopia flexible desde la pubertad $(5,10,19)$. La vigilancia en pacientes sometidos a colectomía con AIR mediante rectoscopia debe ser iniciada 6 meses después de la cirugía y luego $1 \mathrm{vez}$ al año. El seguimiento inicial es el mismo para los pacientes de proctocolectomía con IPAA, con seguimiento posterior cada 2 o 3 años $(18,22)$. Esta periodicidad está condicionada por los hallazgos de la exploración basal o previa (número de adenomas, tamaño e histología de estos), así como la presencia de síntomas o de mutaciones en el gen APC (10).

La alta relación que tiene el estudio exhaustivo de cada paciente con respecto a su correcta terapia y mejor pronóstico demuestra el papel crucial que significa el análisis permanente de guías diagnósticas y constante investigación del tema, ya que alrededor del $30 \%$ de los pacientes con esta enfermedad no posee historia familiar de la misma (4). Esto deriva en que, como en el caso presentado, el diagnóstico se realice en una edad más tardía y en la presencia de síntomas, lo que implica un mayor riesgo de morbimortalidad y desarrollo de CCR (4). Estudios posteriores sobre la genética y epidemiologia de los pacientes sin historia familiar permitirán mejorar las bases diagnósticas y el manejo clínico de los mismos.

\section{REFERENCIAS}

1. Half E, Bercovich D, Rozen P. Familial adenomatous polyposis. Orphanet J Rare Dis. 2009;4:22. https://doi. org/10.1186/1750-1172-4-22.

2. Bisgaard ML, Ripa R, Knudsen AL, Bülow S. Familial adenomatous polyposis patients without an identified APC germline mutation have a severe phenotype. Gut. 2004;53(2):266-70. https://doi.org/10.1136/gut.2003.019042.

3. Cao X, Hong Y,Eu KW, Loi C, Cheah PY.Singapore familial adenomatous polyposis (FAP) patients with classical adenomatous polyposis but undetectable APC mutations have accelerated cancer progression. Am J Gastroenterol. 2006;101(12):28107. https://doi.org/10.1111/j.1572-0241.2006.00842.x.

4. Truta B, Allen BA, Conrad PG, Weinberg V, Miller GA, Pomponio R, et al. A comparison of the phenotype and genotype in adenomatous polyposis patients with and without a family history. Fam Cancer. 2005;4(2):127-33. https://doi.org/10.1007/s10689-004-5814-0.
5. Kobayashi $\mathrm{H}$, Ishida $\mathrm{H}$, Ueno $\mathrm{H}$, Hinoi $\mathrm{T}$, Inoue $\mathrm{Y}$, Ishida $\mathrm{F}$, et al. Association between the age and the development of colorectal cancer in patients with familial adenomatous polyposis: a multi-institutional study. Surg Today. 2017;47(4):470-475. https://doi.org/10.1007/s00595-016-1398-1.

6. Dalavi SB, Vedpalsingh TH, Bankar SS, Ahmed MHS, Bhosale DN. Familial adenomatous polyposis (FAP): a case study and review of literature. J Clin Diagnostic Res. 2015;9(3):PD05-PD06. https://doi.org/10.7860/ JCDR/2015/11636.5696.

7. Kennedy RD, Potter DD, Moir CR, El-Youssef M. The natural history of familial adenomatous polyposis syndrome: A 24-year review of a single center experience in screening, diagnosis, and outcomes. J Pediatr Surg. 2014;49(1):82-86. https://doi.org/10.1016/j.jpedsurg.2013.09.033.

8. Galiatsatos P, Foulkes WD. Familial adenomatous polyposis. Am J Gastroenterol. 2006;101(2):385-98. https://doi. org/10.1111/j.1572-0241.2006.00375.x.

9. Arnason T, Liang WY, Alfaro E, Kelly P, Chung DC, Odze $\mathrm{RD}$, et al. Morphology and natural history of familial adenomatous polyposis-associated dysplastic fundic gland polyps. Histopathology. 2014;65(3):353-362. https://doi. org/10.1111/his.12393.

10. Winawer SJ, Zauber AG, Fletcher RH, Stillman JS, O’Brien MJ, Levin B, et al. Guidelines for Colonoscopy Surveillance After Polypectomy: A Consensus Update by the US MultiSociety Task Force on Colorectal Cancer and the American Cancer Society. Gastroenterology. 2006;130(6):1872-85. https://doi.org/10.1053/j.gastro.2006.03.012.

11. Smith WG, Kern BB. The nature of the mutation in familial multiple polyposis: papillary carcinoma of the thyroid, brain tumors, and familial multiple polyposis. Dis Colon Rectum. 1973;16(4):264-71.

12. Durno C, Monga N, Bapat B, Berk T, Cohen Z, Gallinger S. Does EarlyColectomyIncrease DesmoidRiskinFamilialAdenomatous Polyposis? Clin Gastroenterol Hepatol. 2007;5(10):1190-4. https://doi.org/10.1016/j.cgh.2007.06.010.

13. Tudyka VN, Clark SK. Surgical treatment in familial adenomatous polyposis. Ann Gastroenterol Q Publ Hell Soc Gastroenterol. 2012;25(3):201-6.

14. Win AK, Walters RJ, Buchanan DD, Jenkins MA, Sweet K, Frankel WL, et al. Cancer risks for relatives of patients with serrated polyposis. Am J Gastroenterol. 2012;107(5):770-8. https://doi.org/10.1038/ajg.2012.52.

15. Balaguer Prunés F, Castells i Garangou A. Clínica de alto riesgo de cáncer colorrectal: un nuevo concepto de prevención. Gastroenterol Hepatol Contin. 2007;6(6):289-94.

16. Iwama T, Tamura $\mathrm{K}$, Morita $\mathrm{T}$, Hirai $\mathrm{T}$, Hasegawa $\mathrm{H}$, Koizumi $\mathrm{K}$, et al. A clinical overview of familial adenomatous polyposis derived from the database of the Polyposis Registry of Japan. Int J Clin Oncol. 2004;9(4):308-316. https://doi.org/10.1007/s10147-004-0414-4.

17. Cordero-Fernández C, Garzón-Benavides M, PizarroMoreno A, García-Lozano R, Márquez-Galán JL, López Ruiz T, et al. Gastroduodenal involvement in patients with 
familial adenomatous polyposis. Prospective study of the nature and evolution of polyps: evaluation of the treatment and surveillance methods applied. Eur J Gastroenterol Hepatol. 2009;21(10):1161-7. https://doi.org/10.1097/ MEG.0b013e3283297cf2.

18. Brandão C, Lage J. Management of Patients with Hereditary Colorectal Cancer Syndromes. GE Port J Gastroenterol. 2015;22(5):204-12.https://doi.org/10.1016/j.jpge.2015.06.003.

19. Syngal S, Brand RE, Church JM, Giardiello FM, Hampel HL, Burt RW; et al. ACG Clinical Guideline: Genetic Testing and Management of Hereditary Gastrointestinal Cancer Syndromes. Am J Gastroenterol. 2015;110(2): 22363. https://doi.org/10.1038/ajg.2014.435.
20. Kim B, Giardiello FM. Chemoprevention in familial adenomatous polyposis. Best Pract Res Clin Gastroenterol. 2011;25(45):607-22. https://doi.org/10.1016/j.bpg.2011.08.002.

21. Bresalier RS. Primary chemoprevention of familial adenomatous polyposis with sulindac: More questions than answers. Gastroenterology. 2002;123(1):379-81. https:// doi.org/10.1053/gast.2002.1230379.

22. Navarro M, González S, Iglesias S, Capellá G, RodríguezMoranta F, Blanco I. Síndrome de poliposis hiperplásica: diversidad fenotípica y asociación a cáncer colorrectal. Med Clin (Barc). 2013;141(2):62-6. https://doi.org/10.1016/j. medcli.2012.04.024. 